\begin{tabular}{|c|l|}
\hline Title & HEISENBERG OPERA TORS, INV A RIA NT DOMA INS A ND HEISENBERG EQUA TIONS OF MOTION \\
\hline Author(s) & A rai, A sao \\
\hline Citation & $\begin{array}{l}\text { Reviews in Mathematical Physics, 19(10), 1045-1069 } \\
\text { https://doi.org/10.1142/S0129055X 07003206 }\end{array}$ \\
\hline Issue Date & 2007 \\
\hline Doc URL & http://hdl.handle.net/2115/32287 \\
\hline Rights & $\begin{array}{l}\text { Electronic version of an article published as Reviewsin Mathematical Physics, vol. 19, no. 10, 2007, 1045-1069, } \\
\text { doi:10.1142/S0129055X 07003206 (c) copyright World Scientific Publishing Company }\end{array}$ \\
\hline Type & article (author version) \\
\hline File Information & arai.pdf \\
\hline
\end{tabular}

Instructions for use 


\title{
Heisenberg Operators, Invariant Domains and Heisenberg Equations of Motion
}

\author{
Asao Arai \\ Department of Mathematics \\ Hokkaido University \\ Sapporo 060-0810 \\ Japan \\ E-mail:arai@math.sci.hokudai.ac.jp
}

\begin{abstract}
An abstract operator theory is developed on operators of the form $A_{H}(t):=$ $e^{i t H} A e^{-i t H}, t \in \mathbb{R}$, with $H$ a self-adjoint operator and $A$ a linear operator on a Hilbert space (in the context of quantum mechanics, $A_{H}(t)$ is called the Heisenberg operator of $A$ with respect to $H$ ). The following aspects are discussed: (i) integral equations for $A_{H}(t)$ for a general class of $A$; (ii) a sufficient condition for $D(A)$, the domain of $A$, to be left invariant by $e^{-i t H}$ for all $t \in \mathbb{R}$; (iii) a mathematically rigorous formulation of the Heisenberg equation of motion in quantum mechanics and the uniqueness of its solutions ; (iv) invariant domains in the case where $H$ is an abstract version of Schrödinger and Dirac operators ; (v) applications to Schrödinger operators with matrix-valued potentials and Dirac operators.
\end{abstract}

Keywords: Heisenberg operator; invariant domain ; Heisenberg equation of motion; Schrödinger operator; Dirac operator.

Mathematics Subject Classification 2000: 81Q10, 47N50

\section{Introduction}

Let $\mathcal{H}$ be a complex Hilbert space and $H$ be a self-adjoint operator on $\mathcal{H}$. Then, for a linear operator $A$ on $\mathcal{H}$ and $t \in \mathbb{R}$, one can define a linear operator by

$$
A_{H}(t):=e^{i t H} A e^{-i t H} .
$$

In the context of quantum mechanics, $A_{H}(t)$ is called the Heisenberg operator of $A$ with respect to $H$. If $H$ is the Hamiltonian of a quantum system $\mathrm{S}$ (an operator for the total energy of $\mathrm{S}$ ) and $A$ is a physical quantity of $\mathrm{S}$, then $A_{H}(t)$ describes the time development of $A$ with $t$ being the time. Therefore, in quantum mechanics, it is very important to study Heisenberg operators. It seems, however, that there have been few mathematically rigorous studies of Heisenberg operators in an abstract or a general form, although there 
are ones in concrete models (e.g., $[2,9])$. One of the reasons for this may come from difficulties related to treatments for domains of relevant operators in the case where $H$ and $A$ are unbounded. In this paper, overcoming these difficulties, we present some operator theoretical analyses on the operator $A_{H}(t)$. The point of the paper is to establish a general theory for Heisenberg operators with mathematical rigor and to put a basis for applications.

The present paper is organized as follows. In Section 2 we derive integral equations for $A_{H}(t)$ in the case where $A$ is bounded. This is a preliminary version to the case where $A$ is unbounded. In Section 3 some integral equations for $A_{H}(t)$ are derived in the case where $A$ is a densely defined closed linear operator. In particular, we show that, under some conditions, the domain $D(A)$ of $A$ is an invariant domain of $e^{-i t H}$ for all $t \in \mathbb{R}$. Section 4 is concerned with the so-called Heisenberg equation of motion with respect to $H$. In this case too, some difficulty arises in treating it with mathematical rigor if $H$ is unbounded. But we present a mathematically rigorous form for the Heisenberg equation of motion and discuss the uniqueness of its solutions. In Section 5, using a Weyl representation of the canonical commutation relations with a finite degree of freedom, we introduce an abstract version of Schrödinger operators with matrix-valued potentials and Dirac operators, and prove a theorem on invariant domains and an integral equation for a Heisenberg operator. The last section is devoted to applications of the methods or the results established in the preceding sections to Schrödinger operators with matrix-valued potentials, including relativistic ones, and a class of abstract Dirac operators. As for the Schrödinger operators, we derive a mathematically rigorous version of the "quantum Newton equation" of motion for the position operator. On the other hand, considerations of the abstract Dirac operators clarify a general mathematical structure behind the usual Dirac operator acting in $L^{2}\left(\mathbb{R}^{3} ; \mathbb{C}^{4}\right)([9$, Chapter 4$])$. Applications to models in quantum field theory will be discussed in a separate paper.

\section{Integral equations for $A_{H}(t)$ in the case where $A$ is bounded}

Let $H$ and $A$ be as in Introduction. By the definition of the domain of operator products, we have

$$
D\left(A_{H}(t)\right)=\left\{\psi \in \mathcal{H} \mid e^{-i t H} \psi \in D(A)\right\} .
$$

To treat properly domain problems arising in the analysis of $A_{H}(t)$ in the case where $H$ and $A$ are unbounded, we use some approximation methods. For this purpose we introduce a class of functions:

Definition 2.1 Let $f$ be a real-valued Borel measurable function on $\mathbb{R}$. We say that $f$ is in the class $\mathcal{F}_{+, 1}(\mathbb{R})$ if it is continuous on $[0, \infty)$ and

$$
\begin{aligned}
& f(0)=1, \\
& \sup _{\lambda \geq 0} \lambda|f(\lambda)|<\infty .
\end{aligned}
$$


It is easy to see that

$$
\|f\|_{\infty}:=\sup _{\lambda \geq 0}|f(\lambda)|<\infty, \quad \forall f \in \mathcal{F}_{+, 1}(\mathbb{R}) .
$$

We denote by $\langle\cdot, \cdot\rangle$ and $\|\cdot\|$ the inner product and the norm of $\mathcal{H}$ respectively. The Banach space of all the everywhere defined bounded linear operators on $\mathcal{H}$ is denoted $\mathrm{B}(\mathcal{H})$. The operator norm of $B \in \mathrm{B}(\mathcal{H})$ is written $\|B\|$.

Let $S$ be a densely defined closed linear operator on $\mathcal{H}$. Then, by von Neumann's theorem, $S^{*} S$ is a nonnegative self-adjoint operator (e.g., [8, Theorem X.25]). Hence, for $\varepsilon>0$ and $f \in \mathcal{F}_{+, 1}(\mathbb{R})$, we can define an operator $S_{\varepsilon}$ by

$$
S_{\varepsilon}^{f}:=S f(\varepsilon|S|)
$$

where $|S|:=\left(S^{*} S\right)^{1 / 2}$.

Lemma 2.2 Let $S$ be as above. Then:

(i) $S_{\varepsilon}^{f} \in \mathrm{B}(\mathcal{H})$ and

$$
\lim _{\varepsilon \rightarrow 0} S_{\varepsilon}^{f} \psi=S \psi
$$

for all $\psi \in D(S)$.

(ii) If $S$ is self-adjoint, then $S_{\varepsilon}^{f}$ is self-adjoint and

$$
\begin{aligned}
& \mathrm{S}-\lim _{\varepsilon \rightarrow 0}\left(S_{\varepsilon}^{f}-z\right)^{-1}=(S-z)^{-1}, \quad \forall z \in \mathbb{C} \backslash \mathbb{R}, \\
& \mathrm{S}-\lim _{\varepsilon \rightarrow 0} e^{i t S_{\varepsilon}^{f}}=e^{i t S}, \quad \forall t \in \mathbb{R},
\end{aligned}
$$

where s- lim means strong limit.

Proof. (i) By the polar decomposition, we have $S=U|S|$ with $U$ a partial isometry (e.g., [4, p.334], [7, Theorem VIII.32]). It follows from the functional calculus and (2.3) that $|S| f(\varepsilon|S|)$ is boudned with $D(|S| f(\varepsilon|S|))=\mathcal{H}$. Hence $S_{\varepsilon}^{f} \in \mathrm{B}(\mathcal{H})$. For all $\psi \in$ $D(S)=D(|S|)$, we have

$$
\left\|S_{\varepsilon}^{f} \psi-S \psi\right\|=\||S| f(\varepsilon|S|) \psi-|S| \psi\| .
$$

Using the continuity of $f$ on $[0, \infty)$ together with $(2.2),(2.4)$ and the Lebesgue dominated convergence theorem, one can easily show that the right hand side of (2.9) converges to 0 as $\varepsilon \rightarrow 0$.

(ii) The self-adjointness of $S_{\varepsilon}^{f}$ follows from that $S$ is self-adjoint and $S_{\varepsilon}^{f}=g(S)$ with $g(\lambda)=\lambda f(\varepsilon|\lambda|), \lambda \in \mathbb{R}$. Formulas (2.7) and (2.8) respectively follow from a simple application of a general convergence theorem (e.g., [7, Theorem VIII.25(a), Theorem VIII.21]).

For two linear operators $A$ and $B$, we set

$$
[A, B]:=A B-B A
$$

with $D([A, B]):=D(A B) \cap D(B A)$.

The following lemma is easily proven. 
Lemma 2.3 For all $B \in \mathrm{B}(\mathcal{H}), B_{H}(s)=e^{i s H} B e^{-i s H}$ is in $\mathrm{B}(\mathcal{H})$ and strongly continuous in $s$.

By this lemma, we can define for all $B \in \mathrm{B}(\mathcal{H})$ and $\psi \in \mathcal{H}$ the strong Riemann integral $\int_{0}^{t} e^{i s H} B e^{-i s H} \psi d s \in \mathcal{H}, t \in \mathbb{R}$.

Lemma 2.4 Assume that $H$ and $A$ are in $\mathrm{B}(\mathcal{H})$. Then, for all $t \in \mathbb{R}$ and $\psi \in \mathcal{H}$,

$$
A_{H}(t) \psi=A \psi+\int_{0}^{t} i e^{i s H}[H, A] e^{-i s H} \psi d s .
$$

Proof. Let $\psi \in \mathcal{H}$. Then, under the present assumption, $A_{H}(t) \psi$ is strongly differentiable in $t$ with

$$
\frac{d}{d t} A_{H}(t) \psi=i e^{i t H}[H, A] e^{-i t H} .
$$

Integrating this equation, we obtain (2.11).

The main result of this section is as follows:

Theorem 2.5 Let $A \in \mathrm{B}(\mathcal{H})$. Then, for all $\psi, \phi \in D(H)$,

$$
\left\langle\phi, A_{H}(t) \psi\right\rangle=\langle\phi, A \psi\rangle+i \int_{0}^{t}\left\{\left\langle H \phi, A_{H}(s) \psi\right\rangle-\left\langle\phi, A_{H}(s) H \psi\right\rangle\right\} d s .
$$

Proof. Applying Lemma 2.4 with $H$ replaced by $H_{\varepsilon}^{f}\left(f \in \mathcal{F}_{+, 1}(\mathbb{R})\right)$, we have

$$
\begin{aligned}
& \left\langle\phi, e^{i t H_{\varepsilon}^{f}} A e^{-i t H_{\varepsilon}^{f}} \psi\right\rangle \\
& =\langle\phi, A \psi\rangle \\
& +i \int_{0}^{t}\left\{\left\langle H_{\varepsilon}^{f} e^{-i s H_{\varepsilon}^{f}} \phi, A e^{-i s H_{\varepsilon}^{f}} \psi\right\rangle-\left\langle A^{*} e^{-i s H_{\varepsilon}^{f}} \phi, H_{\varepsilon}^{f} e^{-i s H_{\varepsilon}^{f}} \psi\right\rangle\right\} d s
\end{aligned}
$$

for all $\psi, \phi \in \mathcal{H}$ and $t \in \mathbb{R}$. Applying Lemma 2.2 with $S=H$, we have for all $\psi \in \mathcal{H}$ and $\eta \in D(H)$,

$$
\begin{aligned}
& \text { S- } \lim _{\varepsilon \rightarrow 0} e^{i t H_{\varepsilon}^{f}} A e^{-i t H_{\varepsilon}^{f}} \psi=A_{H}(t) \psi, \quad \text { s- } \lim _{\varepsilon \rightarrow 0} A e^{-i s H_{\varepsilon}^{f}} \psi=A e^{-i s H} \psi, \\
& \text { S- } \lim _{\varepsilon \rightarrow 0} A^{*} e^{-i s H_{\varepsilon}^{f}} \psi=A^{*} e^{-i s H} \psi, \quad \text { s- } \lim _{\varepsilon \rightarrow 0} H_{\varepsilon}^{f} e^{-i s H_{\varepsilon}^{f}} \eta=H e^{-i s H} \eta .
\end{aligned}
$$

Moreover, we have for all $\psi, \phi \in D(H)$

$$
\begin{aligned}
& \left|\left\langle H_{\varepsilon}^{f} e^{-i s H_{\varepsilon}^{f}} \phi, A e^{-i s H_{\varepsilon}} \psi\right\rangle\right| \leq\|H \phi\|\|A\|\|\psi\|\|f\|_{\infty}, \\
& \left|\left\langle A^{*} e^{-i s H_{\varepsilon}^{f}} \phi, H_{\varepsilon}^{f} e^{-i s H_{\varepsilon}^{f}} \psi\right\rangle\right| \leq\left\|A^{*}\right\|\|\phi\|\|H \psi\|\|f\|_{\infty} .
\end{aligned}
$$

Hence we can apply the Lebesgue dominated convergence theorem to the integral on the right hand side of (2.13) to obtain (2.12).

Formula (2.12) may be viewed as an integral equation for $A_{H}(t)$ in a weak sense. 
Corollary 2.6 Let $A \in \mathrm{B}(\mathcal{H})$. Then, for all $\psi \in D(H)$ and $t \in \mathbb{R}, \int_{0}^{t} A_{H}(s) \psi d s \in D(H)$ and

$$
A_{H}(t) \psi=A \psi+i H\left(\int_{0}^{t} A_{H}(s) \psi d s\right)-i \int_{0}^{t} A_{H}(s) H \psi d s .
$$

Proof. Let $\eta(t):=\int_{0}^{t} A_{H}(s) \psi d s$ and $\chi(t):=\int_{0}^{t} A_{H}(s) H \psi d s$. Then, by (2.12), we have for all $\phi \in D(H)\langle H \phi, i \eta(t)\rangle=\left\langle\phi, A_{H}(t) \psi-A \psi+i \chi(t)\right\rangle$. This implies that $i \eta(t) \in D(H)$ and $H(i \eta(t))=A_{H}(t) \psi-A \psi+i \chi(t)$. Thus the desired result follows.

\section{Integral equations for $A_{H}(t)$ with $A$ in a general class and the asymptotic behavior of $A e^{-i t H}$ as $|t| \rightarrow$ $\infty$}

In this section, we consider the case where $A$ is not necessarily bounded. We define a subspace $D_{H, A}$ by

$$
D_{H, A}:=\left\{\psi \in \mathcal{H} \mid e^{i t H} \psi \in D(A), \forall t \in \mathbb{R}\right\}
$$

Obviously

$$
D_{H, A} \subset \cap_{t \in \mathbb{R}} D\left(A_{H}(t)\right) \subset D(A) .
$$

Let $A$ be a densely defined closed linear operator on $\mathcal{H}$. Then, for every $\varepsilon>0, t \in \mathbb{R}$ and $f \in \mathcal{F}_{+, 1}(\mathbb{R})$, we can define a sesquilinear form $q_{\varepsilon, t}^{f}: D(H) \times D(H) \rightarrow \mathbb{C}$ by

$$
\begin{aligned}
q_{\varepsilon, t}^{f}(\phi, \psi):= & i \int_{0}^{t}\left\{\left\langle H e^{-i s H} \phi, A_{\varepsilon}^{f} e^{-i s H} \psi\right\rangle-\left\langle\left(A_{\varepsilon}^{f}\right)^{*} e^{-i s H} \phi, H e^{-i s H} \psi\right\rangle\right\} d s, \\
\phi, \psi \in D(H) &
\end{aligned}
$$

where $A_{\varepsilon}^{f}$ is defined by $(2.5)$ with $S=A$.

Theorem 3.1 Let $A$ be a densely defined closed linear operator on $\mathcal{H}$ and $f \in \mathcal{F}_{+, 1}(\mathbb{R})$. Then, for all $t \in \mathbb{R}, \phi \in D(H)$ and $\psi \in D_{H, A}$,

$$
q_{t}(\phi, \psi):=\lim _{\varepsilon \rightarrow 0} q_{\varepsilon, t}^{f}(\phi, \psi)
$$

exists independently of $f$ and

$$
\left\langle\phi, A_{H}(t) \psi\right\rangle=\langle\phi, A \psi\rangle+q_{t}(\phi, \psi) .
$$

Proof. By (2.12), we have

$$
\left\langle\phi, e^{i t H} A_{\varepsilon}^{f} e^{-i t H} \psi\right\rangle-\left\langle\phi, A_{\varepsilon}^{f} \psi\right\rangle=q_{\varepsilon, t}^{f}(\psi, \phi)
$$

for all $\phi, \psi \in D(H)$ and $t \in \mathbb{R}$. Let $\eta \in D_{H, A}$. Then, by Lemma 2.2, we have

$$
\text { s- } \lim _{\varepsilon \rightarrow 0} A_{\varepsilon}^{f} e^{i \tau H} \eta=A e^{i \tau H} \eta, \quad \forall \tau \in \mathbb{R} .
$$

Hence the left hand side of (3.5) converges to $\left\langle\phi, e^{i t H} A e^{-i t H} \psi\right\rangle-\langle\phi, A \psi\rangle$ as $\varepsilon \rightarrow 0$. Thus the desired assertion follows.

In some cases, the sesquilinear form $q_{t}$ may have more explicit representations. 
Theorem 3.2 Let $A$ be a densely defined closed linear operator on $\mathcal{H}$. Suppose that there exists a subspace $\mathcal{D} \subset D(H) \cap D_{H, A} \cap D_{H, A^{*}}$ such that

$$
\sup _{0 \leq|s| \leq T}\left\|A^{\#} e^{-i s H} \phi\right\|<\infty, \quad \forall T>0, \forall \phi \in \mathcal{D},
$$

where $A^{\#}$ denotes either $A$ or $A^{*}$. Then, for all $\phi, \psi \in \mathcal{D}$ and $t \in \mathbb{R}$,

$$
\left\langle\phi, A_{H}(t) \psi\right\rangle=\langle\phi, A \psi\rangle+i \int_{0}^{t}\left\{\left\langle H e^{-i s H} \phi, A e^{-i s H} \psi\right\rangle-\left\langle A^{*} e^{-i s H} \phi, H e^{-i s H} \psi\right\rangle\right\} d s .
$$

Proof. Under the present assumption, we have for all $\psi, \phi \in \mathcal{D}$ and $s \in \mathbb{R}$

$$
\lim _{\varepsilon \rightarrow 0} A_{\varepsilon}^{f} e^{-i s H} \psi=A e^{-i s H} \psi, \quad \lim _{\varepsilon \rightarrow 0}\left(A_{\varepsilon}^{f}\right)^{*} e^{-i s H} \phi=A^{*} e^{-i s H} \phi .
$$

Let $C_{T, 1}(\psi):=\sup _{0 \leq|s| \leq T}\left\|A e^{-i s H} \psi\right\|$ and $C_{T, 2}(\phi):=\sup _{0 \leq|s| \leq T}\left\|A^{*} e^{-i s H} \phi\right\|$. Then, for all $|s| \leq T$,

$$
\begin{aligned}
& \left|\left\langle H e^{-i s H} \phi, A_{\varepsilon}^{f} e^{-i s H} \psi\right\rangle\right| \leq\|H \phi\|\left\|A e^{-i s H} \psi\right\|\|f\|_{\infty} \leq C_{T, 1}(\psi)\|H \phi\|\|f\|_{\infty}, \\
& \left\langle\left(A_{\varepsilon}^{f}\right)^{*} e^{-i s H} \phi, H e^{-i s H} \psi\right\rangle \mid \leq\|H \psi\|\left\|A^{*} e^{-i s H} \phi\right\|\|f\|_{\infty} \leq C_{T, 2}(\phi)\|H \psi\|\|f\|_{\infty} .
\end{aligned}
$$

Hence, by the Lebesgue dominated convergence theorem, we have for $|t| \leq T$

$$
\lim _{\varepsilon \rightarrow 0} q_{\varepsilon, t}^{f}(\phi, \psi)=i \int_{0}^{t}\left\{\left\langle H e^{-i s H} \phi, A e^{-i s H} \psi\right\rangle-\left\langle A^{*} e^{-i s H} \phi, H e^{-i s H} \psi\right\rangle\right\} d s .
$$

This fact and Theorem 3.1 yield (3.7).

Formula (3.7) can be written as

$$
\left\langle\phi, A_{H}(t) \psi\right\rangle=\langle\phi, A \psi\rangle+i \int_{0}^{t}\left\{\left\langle H \phi, A_{H}(s) \psi\right\rangle-\left\langle A_{H}(s)^{*} \phi, H \psi\right\rangle\right\} d s, \quad \phi, \psi \in \mathcal{D} .
$$

This shows that $A_{H}(t)$ is a solution of the integral equation

$$
\langle\phi, X(t) \psi\rangle=\langle\phi, A \psi\rangle+i \int_{0}^{t}\left\{\langle H \phi, X(s) \psi\rangle-\left\langle X(s)^{*} \phi, H \psi\right\rangle\right\} d s, \quad \phi, \psi \in \mathcal{D},
$$

for an operator-valued function : $t \mapsto X(t)$ with $X(0)=A$.

Corollary 3.3 Let $A$ be a densely defined closed linear operator on $\mathcal{H}$. Suppose that, for some $\alpha \in[1, \infty), A$ and $A^{*}$ are $|H|^{\alpha}$-bounded, i.e., $D\left(|H|^{\alpha}\right) \subset D(A) \cap D\left(A^{*}\right)$ and there exist constants $a_{j} \geq 0, b_{j} \geq 0(j=1,2)$ such that

$$
\|A \psi\| \leq a_{1}\left\||H|^{\alpha} \psi\right\|+b_{1}\|\psi\|, \quad\left\|A^{*} \psi\right\| \leq a_{2}\left\||H|^{\alpha} \psi\right\|+b_{2}\|\psi\|, \quad \forall \psi \in D\left(|H|^{\alpha}\right) .
$$

Then, for all $\phi, \psi \in D\left(|H|^{\alpha}\right)$ and $t \in \mathbb{R}$,

$$
\left\langle\phi, A_{H}(t) \psi\right\rangle=\langle\phi, A \psi\rangle+i \int_{0}^{t}\left\{\left\langle H e^{-i s H} \phi, A e^{-i s H} \psi\right\rangle-\left\langle A^{*} e^{-i s H} \phi, H e^{-i s H} \psi\right\rangle\right\} d s .
$$


Proof. By the present assumption, we have $D\left(|H|^{\alpha}\right) \subset D(H) \cap D_{H, A} \cap D_{H, A^{*}}$. Moreover condition (3.10) implies that, for all $\psi \in D\left(|H|^{\alpha}\right)$ and $|s| \leq T, s \in \mathbb{R}(T>0)$

$$
\left\|A e^{-i s H} \psi\right\| \leq a_{1}\left\||H|^{\alpha} \psi\right\|+b_{1}\|\psi\|, \quad\left\|A^{*} e^{-i s H} \psi\right\| \leq a_{2}\left\||H|^{\alpha} \psi\right\|+b_{2}\|\psi\| .
$$

Hence the assumption of Theorem 3.2 is satisfied with $\mathcal{D}=D\left(|H|^{\alpha}\right)$. Thus the desired result follows.

We next consider conditions under which the integrand of the integral on the right hand side of (3.7) can be written in a simpler form.

Definition 3.4 Let $S$ and $T$ be densely defined linear operators on a Hilbert space $\mathcal{H}$ and $\mathcal{D}$ be a subspace of $\mathcal{H}$ such that $\mathcal{D} \subset D(S) \cap D(T) \cap D\left(S^{*}\right) \cap D\left(T^{*}\right)$. Suppose that, there exists a linear operator $W$ with $D(W)=\mathcal{D}$ such that, for all $\psi, \phi \in \mathcal{D}$

$$
\left\langle S^{*} \psi, T \phi\right\rangle-\left\langle T^{*} \psi, S \phi\right\rangle=\langle\psi, W \phi\rangle .
$$

Then we say that the pair $(S, T)$ has a weak commutator $W$ on $\mathcal{D}$ and write

$$
W=[S, T]_{\mathrm{w}}^{\mathcal{D}} .
$$

Remark 3.1 Since $\mathcal{D}$ is dense, the weak commutator $W$ (if it exists) is uniquely determined.

For a subset $\mathcal{S}$ of $\mathcal{H}$, we denote by $\mathcal{L}(\mathcal{S})$ the subspace algebraically spanned by the vectors in $\mathcal{S}$.

Theorem 3.5 Let $A$ be a densely defined closed linear operator on $\mathcal{H}$. Suppose that there exists a dense subspace $\mathcal{D} \subset D(H) \cap D_{H, A} \cap D_{H, A^{*}}$ such that the pair $(H, A)$ has a weak commutator on the subspace

$$
\mathcal{E}:=\mathcal{L}\left(\left\{e^{-i s H} \psi \mid \psi \in \mathcal{D}, s \in \mathbb{R}\right\}\right),
$$

(note that $\mathcal{E}$ is dense in $\mathcal{H}$ ) and (3.6) holds. Assume that the mapping: $\mathbb{R} \ni s \mapsto$ $[H, A]_{\mathrm{w}}^{\mathcal{E}} e^{-i s H} \psi$ is strongly continuous for all $\psi \in \mathcal{D}$. Then

$$
A_{H}(t) \psi=A \psi+i \int_{0}^{t} e^{i s H}[H, A]_{\mathrm{w}}^{\mathcal{E}} e^{-i s H} \psi d s, \quad t \in \mathbb{R}, \psi \in \mathcal{D} .
$$

Moreover, if

$$
M_{t}(\psi):=\sup _{0 \leq s \leq t}\left\|[H, A]_{\mathrm{w}}^{\mathcal{E}} e^{-i s H} \psi\right\|<\infty, \quad \forall t \geq 0, \forall \psi \in \mathcal{D}
$$

in addition, then

$$
\left\|A_{H}(t) \psi\right\| \leq\|A \psi\|+M_{|t|}(\psi)|t|, \quad \forall t \in \mathbb{R}, \forall \psi \in \mathcal{D} .
$$


Proof. Under the present assumption, for all $\psi \in \mathcal{D}$, the mapping : $s \mapsto e^{i s H}[H, A]_{\mathrm{w}}^{\mathcal{E}}$ $e^{-i s H} \psi(\psi \in \mathcal{D})$ is strongly continuous. Hence we can define a linear operator by

$$
K(t) \psi:=i \int_{0}^{t} e^{i s H}[H, A]_{\mathrm{w}}^{\mathcal{E}} e^{-i s H} \psi d s, \quad \psi \in \mathcal{D}
$$

in the sense of strong integral. Then, by Theorem 3.2, we have

$$
\left\langle\phi, A_{H}(t) \psi\right\rangle=\langle\phi, A \psi\rangle+\langle\phi, K(t) \psi\rangle, \quad \phi, \psi \in \mathcal{D} .
$$

Since $\mathcal{D}$ is dense by the present assumption, (3.13) follows.

Let (3.14) be satisfied. Then, for all $\psi \in \mathcal{D}$,

$$
\|K(t) \psi\| \leq \int_{0}^{|t|}\left\|[H, A]_{\mathrm{w}}^{\mathcal{E}} e^{-i s H} \psi\right\| d s \leq M_{|t|}(\psi)|t| .
$$

Hence (3.15) follows.

Theorem 3.5 implies the following theorem:

Theorem 3.6 Let $A$ be a densely defined closed linear operator on $\mathcal{H}$. Suppose that there exists a dense subspace $\mathcal{D} \subset D(H) \cap D_{H, A} \cap D_{H, A^{*}}$ having the following properties (i)-(iv):

(i) The pair $(H, A)$ has a weak commutator on the subspace $\mathcal{E}$.

(ii) (3.6) holds.

(iii) $\mathcal{D}$ is a core of $A$.

(iv) $[H, A]_{\mathrm{w}}^{\mathcal{E}}$ is bounded.

Then

$$
e^{-i t H} D(A)=D(A), \quad \forall t \in \mathbb{R}
$$

and

$$
\left\|A_{H}(t) \psi\right\| \leq\|A \psi\|+\left\|[H, A]_{\mathrm{w}}^{\mathcal{E}}\right\|\|\psi\||t|, \quad \forall t \in \mathbb{R}, \forall \psi \in D(A) .
$$

Proof. By condition (iv), for all $\psi \in \mathcal{D},[H, A]_{\mathrm{w}}^{\mathcal{E}} e^{-i s H} \psi$ is strongly continuous in $s$ and $M_{|t|}(\psi) \leq C\|\psi\|, \forall t \in \mathbb{R}$ with $C:=\left\|[H, A]_{\mathrm{w}}^{\mathcal{E}}\right\|$. Hence we can apply Theorem 3.5 to the present case. In particular, (3.15) implies that

$$
\left\|A e^{-i t H} \psi\right\| \leq\|A \psi\|+C\|\psi\||t|, \quad \psi \in \mathcal{D} .
$$

Since $\mathcal{D}$ is a core of $A$ by condition (iii), a limiting argument using this inequality shows that, for all $\psi \in D(A), e^{-i t H} \psi \in D(A)$ and (3.18) extends to all $\psi \in D(A)$. Thus the desired reslut follows.

Theorem 3.6 tells us the following: Under the assumption of Theorem 3.6, $D(A)$ is an invariant domain of $e^{-i t H}$ for all $t \in \mathbb{R}$. We remark that this type of theorem has been established in [3, Lemma 2] under a different assumption.

Asymptotic behaviors of $A e^{-t H} \psi(\psi \in D(A))$ as $|t| \rightarrow \infty$ can be derived from Theorem 3.6: 
Corollary 3.7 Under the same assumption as in Theorem 3.6,

$$
\text { w- } \lim _{|t| \rightarrow \infty} \frac{A e^{-i t H}}{t} \psi=0, \quad \forall \psi \in D(A)
$$

where $\mathrm{w}$ - lim means weak limit.

Moreover, for all compact operators $C$ on $\mathcal{H}$,

$$
\lim _{|t| \rightarrow \infty} \frac{C A e^{-i t H}}{t} \psi=0, \quad \forall \psi \in D(A) .
$$

Proof. The idea of proof is same as that of the proof of [9, Corollary 8.7]. By (3.17), we have for all $\psi \in D(A)$

$$
\frac{\left\|A e^{-i t H} \psi\right\|}{|t|} \leq c_{0}, \quad|t| \geq 1
$$

with $c_{0}:=\|A \psi\|+\left\|[H, A]_{\mathrm{w}}^{\mathcal{E}}\right\|\|\psi\|$. Let $\phi \in \mathcal{H}$. Then, for every $\varepsilon>0$, there exists a vector $\phi_{\varepsilon} \in \mathcal{D}$ such that $\left\|\phi_{\varepsilon}-\phi\right\|<\varepsilon$. We have

$$
\left|\left\langle\phi, \frac{A e^{-i t H} \psi}{t}\right\rangle\right| \leq \varepsilon c_{0}+\frac{1}{|t|}\left(\left\|A^{*} \phi_{\varepsilon}\right\| \cdot\|\psi\|\right), \quad|t| \geq 1
$$

Hence

$$
\limsup _{|t| \rightarrow \infty}\left|\left\langle\phi, \frac{A e^{-i t H} \psi}{t}\right\rangle\right| \leq \varepsilon c_{0} .
$$

Since $\varepsilon>0$ is arbitrary, (3.19) follows. Formula (3.20) follows from the well-known fact that a compact operator maps a weakly convergent sequence to a strongly convergent one.

\section{Generalized Heisenberg equations of motion}

Let $H$ be a self-adjoint operator on a Hilbert space $\mathcal{H}$ as before and $\mathcal{D}$ be a dense subspace of $\mathcal{H}$. Then the Heisenberg equation of motion for an operator-valued function : $\mathbb{R} \ni t \mapsto$ $X(t)(X(t)$ is a linear operator on $\mathcal{H})$ with respect to $(H, \mathcal{D})$ is defined by

$$
\frac{d X(t) \psi}{d t}=i[H, X(t)] \psi, \quad \psi \in \mathcal{D}
$$

with the condtion that $\mathcal{D} \subset \cap_{t \in \mathbb{R}} D(H X(t)) \cap D(X(t) H)$ and $X(t) \psi$ is strongly differentiable in $t$.

In the case where $H$ and $X(t)$ are both in $\mathrm{B}(\mathcal{H})$, this definition has no problems and it is easy to see that a $\mathrm{B}(\mathcal{H})$-valued solution $X(t)$ of $(4.1)$ with the initial condition $X(0)=A \in \mathrm{B}(\mathcal{H})$ and $\mathcal{D}=\mathcal{H}$ is unique and given by $X(t)=e^{i t H} A e^{-i t H}$. But, in the case where at least one of $H$ and $X(t)$ is unbounded, the treatment of (4.1) becomes somewhat difficult because of the domain problems and the regularity problem (the non-triviality of strong differentiability of $X(t)$ ). To overcome this difficulty, we propose a weak version of (4.1). 
Definition 4.1 Let $\mathcal{D}$ be a dense subspace of $\mathcal{H}$. We say that an operator-valued function $X(t)$ with $D(X(t))$ dense for all $t \in \mathbb{R}$ obeys a generalized Heisenberg equation of motion with respect to $(H, \mathcal{D})$ if $\mathcal{D} \subset \cap_{t \in \mathbb{R}} D(X(t)) \cap D\left(X(t)^{*}\right) \cap D(H)$ and, for all $\phi, \psi \in \mathcal{D}$, $\langle\phi, X(t) \psi\rangle$ is differentiable in $t \in \mathbb{R}$ with

$$
\frac{d}{d t}\langle\phi, X(t) \psi\rangle=i\left(\langle H \phi, X(t) \psi\rangle-\left\langle X(t)^{*} \phi, H \psi\right\rangle\right), \quad t \in \mathbb{R} .
$$

Proposition 4.2 Let $A$ and $\mathcal{D}$ be as in Theorem 3.2. Then $A_{H}(t)$ is a solution of (4.2) with $X(0)=A$.

Proof. This follows from differentiating (3.8) in $t$.

We next consider the problem on the uniqueness of solutions of (4.2). We introduce a class of operator-valued functions.

Definition 4.3 Let $\mathcal{D}$ be a dense subspace of $\mathcal{H}$ and

$$
\mathcal{F}_{\mathcal{D}}:=\left\{\psi \mid e^{i t H} \psi \in \mathcal{D}, \forall t \in \mathbb{R}\right\} .
$$

We say that an operator-valued function $X(\cdot)$ with $D(X(t))$ dense for all $t \in \mathbb{R}$ is in the set $X_{\mathcal{D}}$ if the following (X.1) and (X.2) hold:

$(\mathrm{X} .1) \mathcal{D} \subset \cap_{t \in \mathbb{R}} D(X(t)) \cap D\left(X(t)^{*}\right) \cap D(H)$.

(X.2) For all $\psi \in \mathcal{F}_{\mathcal{D}}$ and $t \in \mathbb{R}, X(t+s) e^{i t H} \psi$ and $X(s)^{*} e^{i s H} \psi$ are strongly continuous in $s \in \mathbb{R}$ or $X(s) e^{i s H} \psi$ and $X(t+s)^{*} e^{i t H} \psi$ are strongly continuous in $s \in \mathbb{R}$.

Theorem 4.4 Suppose that $\mathcal{F}_{\mathcal{D}}$ is dense in $\mathcal{H}$. Let $X_{0}$ be a densely defined linear operator on $\mathcal{H}$ such that $\mathcal{D} \subset D\left(X_{0}\right) \cap D\left(X_{0}^{*}\right)$. Then the solution $X(\cdot)$ of (4.2) such that $X(\cdot) \in \mathbf{X}_{\mathcal{D}}$ and $X(0)=X_{0}$ is unique on $\mathcal{D}$.

Proof. It is sufficient to prove that, if $X(0) \mid \mathcal{D}=0$, then $X(t) \mid \mathcal{D}=0, \forall t \in \mathbb{R}$. Let $Y(t):=e^{-i t H} X(t) e^{i t H}, t \in \mathbb{R}$ and

$$
L_{\varepsilon}:=\frac{e^{i \varepsilon H}-1}{\varepsilon}, \quad \varepsilon \in \mathbb{R} \backslash\{0\}
$$

We first consider the case where the first condition in (X.2) is satisfied. We have for all $\phi, \psi \in \mathcal{F}_{\mathcal{D}}$

$$
\begin{aligned}
\left\langle\phi, \frac{Y(t+\varepsilon)-Y(t)}{\varepsilon} \psi\right\rangle= & \left\langle X(t+\varepsilon)^{*} e^{i(t+\varepsilon) H} \phi, L_{\varepsilon} e^{i t H} \psi\right\rangle \\
& +\left\langle e^{i t H} L_{\varepsilon} \phi, X(t+\varepsilon) e^{i t H} \psi\right\rangle \\
& +\left\langle e^{i t H} \phi, \frac{X(t+\varepsilon)-X(t)}{\varepsilon} e^{i t H} \psi\right\rangle .
\end{aligned}
$$


This implies that $\langle\phi, Y(t) \psi\rangle$ is differentiable in $t$ with

$$
\begin{aligned}
\frac{d}{d t}\langle\phi, Y(t) \psi\rangle= & i\left\langle X(t)^{*} e^{i t H} \phi, H e^{i t H} \psi\right\rangle-i\left\langle e^{i t H} H \phi, X(t) e^{i t H} \psi\right\rangle \\
& +\left.\frac{d}{d s}\left\langle e^{i t H} \phi, X(s) e^{i t H} \psi\right\rangle\right|_{s=t} .
\end{aligned}
$$

By (4.2), the right hand side is equal to zero. Hence $\langle\phi, Y(t) \psi\rangle=\langle\phi, Y(0) \psi\rangle=0$. Since $\mathcal{F}_{\mathcal{D}}$ is dense, it follows that $Y(t) \psi=0$ for all $\psi \in \mathcal{F}_{\mathcal{D}}$. Hence it follows from the denseness of $\mathcal{F}_{\mathcal{D}}$ that $X(t)^{*} \phi=0$ for all $\phi \in \mathcal{D}$. This implies that $X(t) \mid \mathcal{D}=0$.

In the case where the second condition in (X.2) holds, we need only to rewrite $\left\langle\phi, \varepsilon^{-1}[(Y(t+\varepsilon)-Y(t))] \psi\right\rangle$ as

$$
\begin{aligned}
\left\langle\phi, \frac{Y(t+\varepsilon)-Y(t)}{\varepsilon} \psi\right\rangle= & \left\langle X(t+\varepsilon)^{*} e^{i t H} \phi, L_{\varepsilon} e^{i t H} \psi\right\rangle \\
& +\left\langle e^{i t H} L_{\varepsilon} \phi, X(t+\varepsilon) e^{i(t+\varepsilon) H} \psi\right\rangle \\
& +\left\langle e^{i t H} \phi, \frac{X(t+\varepsilon)-X(t)}{\varepsilon} e^{i t H} \psi\right\rangle
\end{aligned}
$$

and proceed in the same way as in the preceding case.

Corollary 4.5 Let $A$ be a densely defined closed linear operator on $\mathcal{H}$. Suppose that there exists a dense subspace $\mathcal{D} \subset D(H) \cap D_{H, A} \cap D_{H, A^{*}}$ such that the following (i) and (ii) hold:

(i) For all $\psi \in \mathcal{D}, A e^{i t H} \psi$ and $A^{*} e^{i t H}$ are strongly continuous in $t$.

(ii) $\mathcal{F}_{\mathcal{D}}$ is dense in $\mathcal{H}$.

Then $X(t)=A_{H}(t)$ is the unique solution of (4.2) such that $X(\cdot) \in \mathrm{X}_{\mathcal{D}}$ and $X(0) \mid \mathcal{D}=$ $A \mid \mathcal{D}$.

Proof. Condition (i) implies (3.6) in Theorem 3.2. Hence, by Proposition 4.2, $X(t)=$ $A_{H}(t)$ is a solution of (4.2) with $X(0)|\mathcal{D}=A| \mathcal{D}$ and $\mathcal{D} \subset \cap_{t \in \mathbb{R}} D\left(A_{H}(t)\right) \cap D\left(A_{H}(t)^{*}\right) \cap$ $D(H)$. For all $\psi \in \mathcal{F}_{\mathcal{D}}$, we have $A_{H}(t+s) e^{i t H} \psi=e^{i(t+s) H} A e^{-i s H} \psi$ and $A_{H}(s)^{*} e^{i s H} \psi=$ $e^{i s H} A^{*} \psi$. Hence $A_{H}(t+s) e^{i t H} \psi$ and $A_{H}(s)^{*} e^{i s H} \psi$ are strongly continuous in $s$. Therefore $A_{H} \in \mathrm{X}_{\mathcal{D}}$. Thus, by Theorem 4.4, the uniqueness of $A_{H}(\cdot)$ follows.

Remark 4.1 In applications, Corollary 4.5 can be used as follows: Suppose that one finds an operator $X(t)$ satisfying (4.2) with $X(\cdot) \in \mathrm{X}_{\mathcal{D}}$ and $X(0)|\mathcal{D}=A| \mathcal{D}$ and that $X(t)$ has an explicit representation. Then, by Corollary $4.5, A_{H}(t)|\mathcal{D}=X(t)| \mathcal{D}$, which gives an explicit representation for $A_{H}(t) \mid \mathcal{D}$. Elementary examples in quantum physics for which this method works are as follows: (i) quantum particles in $\mathbb{R}^{n}$ with potentials of quadratic polynomials, including harmonic oscillators ; (ii) quasi free quantum field models. But we do not discuss them here. 


\section{Weyl Representations of canonical commutation relations and invariant domains}

In this section we show that there is a general mathematical structure for existence of invariant domains of the strongly continuous one-parameter unitary group generated by an abstract self-adjoint operator which is made from the Weyl representation of canonical commutation relations (CCR) with a finite degree of freedom.

Let $\mathcal{H}$ be a complex Hilbert space and $\left\{Q_{j}, P_{j} \mid j=1, \cdots, n\right\}$ be a set of self-adjoint operators on $\mathcal{H}$ obeying the following conditions:

(i) For all $s, t \in \mathbb{R}$ and $j, k=1, \cdots, n$

$$
e^{i t Q_{j}} e^{i s Q_{k}}=e^{i s Q_{k}} e^{i t Q_{j}}, \quad e^{i t P_{j}} e^{i s P_{k}}=e^{i s P_{k}} e^{i t P_{j}} .
$$

(ii) For all $j, k=1, \cdots, n$ and $s, t \in \mathbb{R}$

$$
e^{i t Q_{j}} e^{i s P_{k}}=e^{-i t s \delta_{j k}} e^{i s P_{k}} e^{i t Q_{j}} .
$$

The set $\left\{Q_{j}, P_{j} \mid j=1, \cdots, n\right\}$ is called a Weyl representation of the CCR with $n$ degrees of freedom. It is well known that, if $\mathcal{H}$ is separable, then $\left\{Q_{j}, P_{j} \mid j=1, \cdots, n\right\}$ is unitarily equivalent to a direct sum of the Schrödinger representation of the CCR with $n$ degrees of freedom [5]. But we work with $\left\{Q_{j}, P_{j} \mid j=1, \cdots, n\right\}$, since it is suitable for analysis of general mathematical structures that the Weyl representation of CCR has.

Here we recall the definition of strong commutativity on self-adjoint operators. For a self-adjoint operator $S$, we denote by $E_{S}(\cdot)$ its spectral measure.

Definition 5.1 Two self-adjoint operators $S$ and $T$ on a Hilbert space are said to strongly commute if their spectral measures commute, i.e., $E_{S}(J) E_{T}(K)=E_{T}(K) E_{S}(J)$ for all Borel sets $J, K \subset \mathbb{R}$. In this case, we say also that $S$ strongly commutes with $T$.

A set $\left\{S_{j}\right\}_{j=1}^{n}(n \in \mathbb{N})$ of self-adjoint operators on a Hilbert space is said to be strongly commuting if, for each pair $(j, k)$ with $j \neq k(j, k=1, \cdots, n), S_{j}$ and $S_{k}$ strongly commute.

Lemma 5.2 Let $Q_{j}$ and $P_{j}$ be as above. Then:

(i) $\left\{Q_{j}\right\}_{j=1}^{n}$ is strongly commuting.

(ii) $\left\{P_{j}\right\}_{j=1}^{n}$ is strongly commuting.

(iii) For all $j, k=1, \cdots, n$ with $j \neq k, P_{j}$ and $Q_{k}$ strongly commute.

(iv) For all $t \in \mathbb{R}$ and $k, j=1, \cdots, n$

$$
e^{i t Q_{k}} D\left(P_{j}\right)=D\left(P_{j}\right)
$$

and

$$
e^{i t Q_{k}} P_{j} e^{-i t Q_{k}}=P_{j}-\delta_{j k} t .
$$


Proof. The facts of (i) and (ii) follow from a general theorem (e.g.,[7, Theorem VIII.13]).

Formula (5.1) with $j \neq k$ gives $e^{i t Q_{j}} e^{i s P_{k}}=e^{i s P_{k}} e^{i t Q_{j}}$ for all $s, t \in \mathbb{R}$. Hence, by a general theorem ([7, Theorem VIII.13]) again, the desired result follows.

To prove (iii), let $\psi \in D\left(P_{j}\right)$. Then, by (5.1), $e^{i t Q_{k}} e^{i s t \delta_{j k}} e^{i s P_{j}} \psi=e^{i s P_{j}} e^{i t Q_{k}} \psi$ for all $s, t \in \mathbb{R}$. The vector-valued function $e^{i s P_{j}} \psi$ of $s$ is strongly differentiable in $s$. Hence the left hand side is strongly differentiable in $s$. Therefore the right hand side is strongly differentiable in $s$. Thus $e^{i t Q_{k}} \psi \in D\left(P_{j}\right)$ and

$$
e^{i t Q_{k}} t \delta_{j k} \psi+e^{i t Q_{k}} P_{j} \psi=P_{j} e^{i t Q_{k}} \psi \text {. }
$$

Hence $e^{i t Q_{k}} D\left(P_{j}\right) \subset D\left(P_{j}\right)$. This implies (5.2). Then (5.3) follows from (5.4).

By the strong commutativity of $Q:=\left\{Q_{j}\right\}_{j=1}^{n}$ [Lemma 5.2)-(i)], there exists a unique $n$-dimensional spectral measure $\hat{E}_{Q}$, called the joint spectral measure of $Q$, such that, for all Borel sets $B_{j}$ in $\mathbb{R}(j=1, \cdots, n)$

$$
\hat{E}_{Q}\left(B_{1} \times \cdots \times B_{n}\right)=E_{Q_{1}}\left(B_{1}\right) \cdots E_{Q_{n}}\left(B_{n}\right) .
$$

Then one can develop the functional calculus with respect to $\hat{E}_{Q}$. Namely, for each Borel measurable function $F$ on $\mathbb{R}^{n}$, we can define a linear operator $F(Q)$ on $\mathcal{H}$ by

$$
F(Q):=\int_{\mathbb{R}^{n}} F(\lambda) d \hat{E}_{Q}(\lambda)
$$

i.e.,

$$
\begin{aligned}
& D(F(Q))=\left\{\left.\psi \in \mathcal{H}\left|\int_{\mathbb{R}^{n}}\right| F(\lambda)\right|^{2} d\left\|\hat{E}_{Q}(\lambda) \psi\right\|^{2}<\infty\right\}, \\
& \langle\phi, F(Q) \psi\rangle=\int_{\mathbb{R}^{n}} F(\lambda) d\left\langle\phi, \hat{E}_{Q}(\lambda) \psi\right\rangle, \phi \in \mathcal{H}, \psi \in D(F(Q)) .
\end{aligned}
$$

If $F$ is real-valued such that $\hat{E}_{Q}\left(\left\{\lambda \in \mathbb{R}^{n}|| F(\lambda) \mid=+\infty\right\}\right)=0$, then $F(Q)$ is self-adjoint. Similarly $P:=\left\{P_{j}\right\}_{j=1}^{n}$ has the joint spectral measure $\hat{E}_{P}$. In what follows, we define, using $\hat{E}_{Q}$ and $\hat{E}_{P}$, an abstract version of quantum mechanical Hamiltonians, including the usual Schrödinger type operators with matrix-valued potentials and Dirac type ones.

For $\ell=1, \cdots, L(L \in \mathbb{N})$, let $T_{\ell}: \mathbb{R}^{n} \rightarrow \mathbb{R} ; \mathbb{R}^{n} \ni \lambda \mapsto T_{\ell}(\lambda) \in \mathbb{R}$ be a continuously differentiable function satisfying the following conditions (T.1) and (T.2):

(T.1) For each $j=1, \cdots, n$ and $\ell=1, \cdots, L$, there exist positive constants $a_{\ell j}, b_{\ell j}$ such that, for all sufficiently small $|t|(t \in \mathbb{R})$,

$$
\left|T_{\ell}\left(\lambda+t \mathbf{e}_{j}\right)\right|^{2} \leq a_{\ell j}\left|T_{\ell}(\lambda)\right|^{2}+b_{\ell j}, \forall \lambda \in \mathbb{R}^{n},
$$

where $\mathbf{e}_{j}:=(0, \cdots, 0, \stackrel{j \text {-th }}{1}, 0, \cdots, 0) \in \mathbb{R}^{n}(j=1, \cdots, n)$.

(T.2) For $j=1, \cdots, n$ and $\ell=1, \cdots, L$, there exist positive constants $c_{\ell j}, d_{\ell j}$ such that, for all sufficiently small $|t|$,

$$
\left|\partial_{j} T_{\ell}\left(\lambda+t \mathbf{e}_{j}\right)\right|^{2} \leq c_{\ell j}\left|T_{\ell}(\lambda)\right|^{2}+d_{\ell j}, \forall \lambda \in \mathbb{R}^{n},
$$

where $\left(\partial_{j} T_{\ell}\right)(\lambda):=\partial T_{\ell}(\lambda) / \partial \lambda_{j}$. 
Let $\mathcal{K}$ be a Hilbert space and $\left\{A_{\ell}, B_{\ell} \mid \ell=1, \cdots, L\right\}$ be a set of bounded self-adjoint operators on $\mathcal{K}\left(A_{\ell}, B_{\ell} \in \mathrm{B}(\mathcal{K})\right)$. Let $V_{\ell}(\ell=1, \cdots, L)$ be a real-valued Borel measurable function on $\mathbb{R}^{n}$ such that $\hat{E}_{Q}\left(\left\{\lambda \in \mathbb{R}^{n}|| V_{\ell}(\lambda) \mid=+\infty\right\}\right)=0$. Then $T_{\ell}(P)$ and $V_{\ell}(Q)$ are self-adjoint. We define an linear operator $H$ acting in $\mathcal{K} \otimes \mathcal{H}$ as follows:

$$
H:=\sum_{\ell=1}^{L}\left[A_{\ell} \otimes T_{\ell}(P)+B_{\ell} \otimes V_{\ell}(Q)\right] .
$$

We set

$$
H_{0}(P):=\sum_{\ell=1}^{L} A_{\ell} \otimes T_{\ell}(P), \quad H_{\mathrm{I}}:=\sum_{\ell=1}^{L} B_{\ell} \otimes V_{\ell}(Q)
$$

Hence

$$
H=H_{0}(P)+H_{\mathrm{I}}
$$

We assume the following:

\section{Hypothesis $(\mathrm{H})$}

(H.1) Each $A_{\ell}$ has a bounded inverse $A_{\ell}^{-1} \in \mathrm{B}(\mathcal{H})$.

(H.2) The operator $H$ is self-adjoint.

The main result of this section is the following theorem:

Theorem 5.3 Under Hypothesis (H), the following (i) and (ii) hold:

(i) For all $t \in \mathbb{R}$ and $j=1, \cdots, n$,

$$
e^{-i t H} D\left(I \otimes Q_{j}\right) \cap D(H)=D\left(I \otimes Q_{j}\right) \cap D(H),
$$

where $I$ denotes identity. Moreover, for all $\psi \in D\left(I \otimes Q_{j}\right) \cap D(H)$,

$$
e^{i t H}\left(I \otimes Q_{j}\right) e^{-i t H} \psi=I \otimes Q_{j} \psi+\sum_{\ell=1}^{L} \int_{0}^{t} e^{i \tau H} A_{\ell} \otimes\left(\partial_{j} T_{\ell}\right)(P) e^{-i \tau H} \psi d \tau,
$$

where the integral on the right hand side is taken in the strong sense.

(ii) If each $\partial_{j} T_{\ell}(j=1, \cdots, n, \ell=1, \cdots, L)$ is bounded and $D\left(I \otimes Q_{j}\right) \cap D(H)$ is a core for $I \otimes Q_{j}$ in addition, then

$$
e^{-i t H} D\left(I \otimes Q_{j}\right)=D\left(I \otimes Q_{j}\right), \quad \forall t \in \mathbb{R}
$$

and (5.10) holds for all $\psi \in D\left(I \otimes Q_{j}\right)$.

To prove this theorem, we need some lemmas. It follows from (H.2) and the closed graph theorem that there exists a constant $C>0$ such that

$$
\left\|A_{\ell} \otimes T_{\ell}(P) \psi\right\| \leq C(\|H \psi\|+\|\psi\|), \quad \forall \psi \in D(H), \ell=1, \cdots, L .
$$

Condition (H.1) implies that

$$
\left\|I \otimes T_{\ell}(P) \psi\right\| \leq\left\|A_{\ell}^{-1}\right\|\left\|A_{\ell} \otimes T_{\ell}(P) \psi\right\|, \quad \forall \psi \in D\left(A_{\ell} \otimes T_{\ell}(P)\right), \ell=1, \cdots, L .
$$

Hence

$$
\left\|I \otimes T_{\ell}(P) \psi\right\| \leq\left\|A_{\ell}^{-1}\right\| C(\|H \psi\|+\|\psi\|), \quad \forall \psi \in D(H), \ell=1, \cdots, L .
$$


Lemma 5.4 Let $U$ be a unitary operator from a Hilbert space $\mathcal{H}_{1}$ to a Hilbert space $\mathcal{H}_{2}$ and $R_{j}(j=1, \cdots, N, N \in \mathbb{N})$ be a linear operator from $\mathcal{H}_{1}$ to $\mathcal{H}_{2}$ with domain $D\left(R_{j}\right) \subset \mathcal{H}_{1}$. Then the operator equality

$$
U\left(\sum_{j=1}^{N} R_{j}\right) U^{-1}=\sum_{j=1}^{N} U R_{j} U^{-1}
$$

holds.

Proof. We need only to show that $D\left(U\left(\sum_{j=1}^{N} R_{j}\right) U^{-1}\right)=\cap_{j=1}^{N} D\left(U R_{j} U^{-1}\right)$. But this is straightforward.

Lemma 5.5 For all $t \in \mathbb{R}, e^{i t\left(I \otimes Q_{j}\right)} H_{\mathrm{I}} e^{-i t\left(I \otimes Q_{j}\right)}=H_{\mathrm{I}}$ (operator equality).

Proof. For all $\psi \in \mathcal{K} \hat{\otimes} D\left(V_{\ell}(Q)\right)$ ( $\hat{\otimes}$ denotes algebraic tensor product), $e^{i t\left(I \otimes Q_{j}\right)} B_{\ell} \otimes$ $V_{\ell}(Q) e^{-i t\left(I \otimes Q_{j}\right)} \psi=B_{\ell} \otimes V_{\ell}(Q) \psi(\ell=1, \cdots, L)$. Hence $B_{\ell} \otimes V_{\ell}(Q) \subset e^{i t\left(I \otimes Q_{j}\right)} B_{\ell} \otimes$ $V_{\ell}(Q) e^{-i t\left(I \otimes Q_{j}\right)}$. Since the both sides are self-adjoint, we have $B_{\ell} \otimes V_{\ell}(Q)=e^{i t\left(I \otimes Q_{j}\right)} B_{\ell} \otimes$ $V_{\ell}(Q) e^{-i t\left(I \otimes Q_{j}\right)}$. By this relation and Lemma 5.4, we obtain the desired result.

Lemma 5.6 For all $t \in \mathbb{R}, e^{i t\left(I \otimes Q_{j}\right)} H_{0}(P) e^{-i t\left(I \otimes Q_{j}\right)}=H_{0}\left(P-t \mathbf{e}_{j}\right)$ (operator equality).

Proof. By (5.3) and the functional calculus, we have $e^{i t Q_{j}} T_{\ell}(P) e^{-i t Q_{j}}=T_{\ell}\left(P-t \mathbf{e}_{j}\right)$, which implies that $e^{i t\left(I \otimes Q_{j}\right)} A_{\ell} \otimes T_{\ell}(P) e^{-i t\left(I \otimes Q_{j}\right)}=A_{\ell} \otimes T_{\ell}\left(P-t \mathbf{e}_{j}\right)$. This relation and Lemma 5.4 give the desired result.

Lemma 5.7 There exists a constant $\delta>0$ such that, for all $|t|<\delta$,

$$
D\left(I \otimes T_{\ell}\left(P-t \mathbf{e}_{j}\right)\right)=D\left(I \otimes T_{\ell}(P)\right), \quad j=1, \cdots, n, \ell=1, \cdots, L,
$$

and

$$
\begin{gathered}
\left\|\left[I \otimes T_{\ell}\left(P-t \mathbf{e}_{j}\right)-I \otimes T_{\ell}(P)\right] \psi\right\| \leq \quad K|t|(\|H \psi\|+\|\psi\|), \quad \psi \in D(H), \\
j=1, \cdots, n, \ell=1, \cdots, L,
\end{gathered}
$$

where $K>0$ is a constant independent of $t$.

Proof. The first half of the lemma follows from condition (T.1) and the functional calculus. By the mean value theorem and condition (T.2), we have for all sufficiently small $|t|$

$$
\left|T_{\ell}\left(\lambda-t \mathbf{e}_{j}\right)-T_{\ell}(\lambda)\right|^{2} \leq C_{1}^{2}|t|^{2}\left(\left|T_{\ell}(\lambda)\right|^{2}+1\right),
$$

where $C_{1}>0$ is a constant. Hence

$$
\left\|\left[T_{\ell}\left(P-t \mathbf{e}_{j}\right)-T_{\ell}(P)\right] \phi\right\| \leq C_{1}|t|\left(\left\|T_{\ell}(P) \psi\right\|+\|\phi\|\right), \quad \phi \in D\left(T_{\ell}(P)\right) .
$$

By this estimate and (5.13), we obtain (5.15).

For $j=1, \cdots, n$ and $t \in \mathbb{R}$, we define

$$
H_{j}(t):=H_{0}\left(P-t \mathbf{e}_{j}\right)+H_{\mathrm{I}} .
$$


Lemma 5.8 For $j=1, \cdots, n$ and all sufficiently small $|t|, H_{j}(t)$ is self-adjoint with $D\left(H_{j}(t)\right)=D(H)$ and

$$
e^{i t\left(I \otimes Q_{j}\right)} H e^{-i t\left(I \otimes Q_{j}\right)}=H_{j}(t) .
$$

In particular, for all $t \in \mathbb{R}, e^{-i t\left(I \otimes Q_{j}\right)} D(H)=D(H)$.

Proof. We can write

$$
H_{j}(t)=H+\sum_{\ell=1}^{L} W_{j \ell}(t)
$$

where $W_{j \ell}(t):=A_{\ell} \otimes T_{\ell}\left(P-t \mathbf{e}_{j}\right)-A_{\ell} \otimes T_{\ell}(P)$. Let $\psi \in D(H)$. Then, by Lemma 5.7, we obtain

$$
\left\|W_{j \ell} \psi\right\| \leq C_{2}|t|(\|H \psi\|+\|\psi\|),
$$

where $C_{2}>0$ is a constant independent of $t$. Hence, by the Kato-Rellich theorem, for all $|t|$ such that $L C_{2}|t|<1, H_{j}(t)$ is self-adjoint with $D\left(H_{j}(t)\right)=D(H)$. Formula (5.17) follows from Lemmas 5.5 and 5.6.

The result of the preceding paragraph implies that, for all sufficiently small $|t|$,

$$
e^{-i t\left(I \otimes Q_{j}\right)} D(H)=D(H) .
$$

But, by using the group property of $e^{-i t\left(I \otimes Q_{j}\right)}$ in $t$, one can show that this holds for all $t \in \mathbb{R}$.

Lemma 5.9 Let $A$ and $B$ be self-adjoint operators on $\mathcal{H}$ such that $A+B$ is self-adjoint and $B$ is $A$-bounded. Then, for all $\psi \in D(A)$ and $t \in \mathbb{R}$,

$$
e^{-i t(A+B)} \psi=e^{-i t A} \psi-i \int_{0}^{t} e^{-i(t-s)(A+B)} B e^{-i s A} \psi d s,
$$

where the integral on the right hand side is taken in the sense of strong Riemann integral.

Proof. This lemma is probably well known. But, for completeness, we give a proof. We first consider the case where both $A$ and $B$ are in $\mathrm{B}(\mathcal{H})$. Let $\phi(t)=e^{-i t A} \psi-$ $i \int_{0}^{t} e^{-i(t-s)(A+B)} B e^{-i s A} \psi d s$. Then it is easy to show that $\phi(t)$ is strongly differentiable in $t$ and

$$
i \frac{d}{d t} \phi(t)=(A+B) \phi(t) .
$$

Hence $\phi(t)=e^{-i(A+B) t} \phi(0)=e^{-i(A+B) t} \psi$. Therefore (5.18) holds.

We next consider the case where $A$ is not necessarily bounded and $B \in \mathrm{B}(\mathcal{H})$. Let $A_{\varepsilon}^{f}$ be the operator $S_{\varepsilon}^{f}$ defined by (2.5) with $S$ replaced by $A$. Then, by the preceding result, we have

$$
e^{-i t\left(A_{\varepsilon}^{f}+B\right)} \psi=e^{-i t A_{\varepsilon}^{f}} \psi-i \int_{0}^{t} e^{-i(t-s)\left(A_{\varepsilon}^{f}+B\right)} B e^{-i s A_{\varepsilon}^{f}} \psi d s .
$$

For all $\phi \in D(A), \lim _{\varepsilon \rightarrow 0}\left(A_{\varepsilon}+B\right) \phi=(A+B) \phi$. Hence, by a general convergence theorem, $\lim _{\varepsilon \rightarrow 0} e^{-i t\left(A_{\varepsilon}^{f}+B\right)} \psi=e^{-i t(A+B)} \psi$. Moreover

$$
\begin{aligned}
& \left\|\int_{0}^{t} e^{-i(t-s)\left(A_{\varepsilon}^{f}+B\right)} B e^{-i s A_{\varepsilon}^{f}} \psi d s-\int_{0}^{t} e^{-i(t-s)(A+B)} B e^{-i s A} \psi d s\right\| \\
& \leq \int_{0}^{|t|}\|B\|\left\|e^{-i s A_{\varepsilon}^{f}} \psi-e^{-i s A} \psi\right\| d s+\int_{0}^{|t|}\left\|\left(e^{-i(t-s)\left(A_{\varepsilon}^{f}+B\right)}-e^{-i(t-s)(A+B)}\right) B e^{-i s A} \psi\right\| d s .
\end{aligned}
$$


Hence, using the Lebesgue dominated convergence theorem, we see that the right hand side converges to 0 as $\varepsilon \rightarrow 0$. Thus, taking the limit $\varepsilon \rightarrow 0$ in (5.19), we obtain (5.18).

Finally we consider the case where both $A$ and $B$ satisfy the assumption of the present lemma. By the result of the preceding paragraph, we have

$$
e^{-i t\left(A+B_{\varepsilon}^{f}\right)} \psi=e^{-i t A} \psi-i \int_{0}^{t} e^{-i(t-s)\left(A+B_{\varepsilon}^{f}\right)} B_{\varepsilon}^{f} e^{-i s A} \psi d s .
$$

Since $B$ is $A$-bounded, it follows that $D(A) \subset D(B)$ and $e^{-i s A} \phi \in D(A) \subset D(B)$ for all $\phi \in D(A)$ and $s \in \mathbb{R}$. Hence

$$
\lim _{\varepsilon \rightarrow 0} B_{\varepsilon}^{f} e^{-i s A} \psi=B e^{-i s A} \psi
$$

Using the $A$-boundedness of $B$, one can show that $e^{-i(t-s)(A+B)} B e^{-i s A} \psi$ is strongly continuous in $s \in \mathbb{R}$. Hence, in the same way as in the preceding paragraph, we can show that taking the limit $\varepsilon \rightarrow 0$ in (5.20) yields (5.18).

\section{Proof of Theorem 5.3}

Throughout the proof, we set $\hat{Q}_{j}:=I \otimes Q_{j}$.

(i) We can write

$$
H_{j}(s)=H+C(s)
$$

with $C(s):=H_{0}\left(P-s \mathbf{e}_{j}\right)-H_{0}(P)$, where $|s|(s \in \mathbb{R})$ is sufficiently small. Then, by Lemma 5.8 and the functional calculus, we have for all Borel measurable function $u$ on $\mathbb{R}$

$$
e^{i s \hat{Q}_{j}} u(H) e^{-i s \hat{Q}_{j}}=u(H+C(s)) .
$$

Hence, taking $u(\lambda)=e^{-i t \lambda}, \lambda \in \mathbb{R}$, we obtain for all $t \in \mathbb{R}$ and sufficiently small $|s|$

$$
e^{i s \hat{Q}_{j}} e^{-i t H}=e^{-i t(H+C(s))} e^{i s \hat{Q}_{j}} .
$$

Hence, for all $\psi \in D\left(\hat{Q}_{j}\right)(s \neq 0)$,

$$
\begin{aligned}
\frac{\left(e^{i s \hat{Q}_{j}}-1\right)}{s} e^{-i t H} \psi= & e^{-i t(H+C(s))}\left(\frac{\left(e^{i s \hat{Q}_{j}}-1\right) \psi}{s}-i \hat{Q}_{j} \psi\right)+e^{-i t(H+C(s))} i \hat{Q}_{j} \psi \\
& +\frac{e^{-i t(H+C(s))}-e^{-i t H}}{s} \psi
\end{aligned}
$$

We have

$$
\lim _{s \rightarrow 0}\left(\frac{\left(e^{i s \hat{Q}_{j}}-1\right) \psi}{s}-i \hat{Q}_{j} \psi\right)=0 .
$$

Since $e^{-i t(H+C(s))}$ is unitary, it follows that

$$
\lim _{s \rightarrow 0} e^{-i t(H+C(s))}\left(\frac{\left(e^{i s \hat{Q}_{j}}-1\right) \psi}{s}-i \hat{Q}_{j} \psi\right)=0 .
$$


By Lemma 5.7, $C(s)$ is $H$-bounded and $\lim _{s \rightarrow 0} C(s) \eta=0$ for all $\eta \in D(H)$. Hence, by general convergence theorems [7, VIII.21, VIII.25(a)], we have

$$
\lim _{s \rightarrow 0} e^{-i t(H+C(s))} \phi=e^{-i t H} \phi, \quad \phi \in \mathcal{H} .
$$

Hence

$$
\lim _{s \rightarrow 0} e^{-i t(H+C(s))} i \hat{Q}_{j} \psi=i e^{-i t H} \hat{Q}_{j} \psi
$$

By Lemma 5.9, we have

$$
\frac{e^{-i t(H+C(s))}-e^{-i t H}}{s} \psi=-i \int_{0}^{t} e^{-i(t-\tau)(H+C(s))} \frac{C(s)}{s} e^{-i \tau H} \psi d \tau .
$$

By the functional calculus, we can show that, for all $\phi \in D(H), \lim _{s \rightarrow 0} C(s) \phi / s=$ $-\sum_{\ell=1}^{L} A_{\ell} \otimes\left(\partial_{j} T_{\ell}\right)(P) \phi$ and $\|C(s) \phi / s\| \leq a\|(H+1) \phi\|$, where $a$ is a positive constant independent of $s$. Hence it follows that

$$
\left.\lim _{s \rightarrow 0} \frac{e^{-i t(H+C(s))}-e^{-i t H}}{s} \phi=i \sum_{\ell=1}^{L} \int_{0}^{t} e^{-i(t-\tau) H} A_{\ell} \otimes\left(\partial_{j} T_{\ell}\right)(P)\right) e^{-i \tau H} \phi d \tau, \quad \forall \phi \in D(H) .
$$

Putting these results into (5.21), one can conclude that, for all $\psi \in D\left(\hat{Q}_{j}\right) \cap D(H)$, $e^{-i t H} \psi \in D\left(\hat{Q}_{j}\right) \cap D(H)$ and

$$
\left.\hat{Q}_{j} e^{-i t H} \psi=e^{-i t H} \hat{Q}_{j} \psi+\sum_{\ell=1}^{L} \int_{0}^{t} e^{-i(t-\tau) H} A_{\ell} \otimes\left(\partial_{j} T_{\ell}\right)(P)\right) e^{-i \tau H} \psi d \tau .
$$

Hence (5.9) and (5.10) follow.

(ii) In this case, each $A_{\ell} \otimes\left(\partial_{j} T_{\ell}\right)(P)$ is in $\mathrm{B}(\mathcal{K} \otimes \mathcal{H})$. By using this property and that $D\left(\hat{Q}_{j}\right) \cap D(H)$ is a core of $\hat{Q}_{j}$, one can extend, by a simple limiting argument, (5.9) to all $\psi \in D\left(\hat{Q}_{j}\right)$, at the same time, showing that, for all $\psi \in D\left(\hat{Q}_{j}\right)$ and all $t \in \mathbb{R}$, $e^{-i t H} \psi \in D\left(\hat{Q}_{j}\right)$. Thus the desired result follows.

Remark 5.1 The operator $H$ is symmetric in the exchange of $\left(A_{\ell}, T_{\ell}(P)\right)$ and $\left(B_{\ell}, V_{\ell}(Q)\right)$ $(\ell=1, \cdots, L)$. Hence Theorem 5.3 holds with $Q_{j}\left(\right.$ resp. $\left.A_{\ell}, T_{\ell}(P)\right)$ replaced by $-P_{j}$ (resp. $\left.B_{\ell}, V_{\ell}(Q)\right)$ under the assumption for $\left(A_{\ell}, T_{\ell}(P)\right)(\ell=1, \cdots, L)$ replaced by $\left(B_{\ell}, V_{\ell}(Q)\right)$ $(\ell=1, \cdots, L)$ (note that, in the present case, $-P_{j}$ plays the role of $Q_{j}$ in Theorem 5.3).

\section{Applications to Schrödinger and Dirac Operators}

In this section we apply the results in the preceding section to Schrödinger and Dirac operators with operator-valued potentials. 


\subsection{Schrödnger operators}

Let $\left\{q_{j}, p_{j}\right\}_{j=1}^{n}$ be the Schrödinger representaton of the CCR with $n$ degrees of freedom, i.e., $q_{j}$ is the multiplication operator by the $j$-th coordinate variable $x_{j}$ in $x=\left(x_{1}, \cdots, x_{n}\right) \in$ $\mathbb{R}^{n}$ acting in $L^{2}\left(\mathbb{R}^{n}\right)$ and $p_{j}:=-i D_{j}$, where $D_{j}$ is the generalized partial differential operator in $x_{j}$ acting in $L^{2}\left(\mathbb{R}^{n}\right)$. It is well-known (or easy to see) that $\left\{q_{j}, p_{j}\right\}_{j=1}^{n}$ is a Weyl representation. In the context of quantum mechanics, $q_{j}$ and $p_{j}$ are called the $j$-th position operator and the $j$-th momentum operator respectively. We consider the following case in the notation of the preceding section:

$$
\begin{aligned}
& \mathcal{H}=L^{2}\left(\mathbb{R}^{n}\right), \quad Q_{j}=q_{j}, P_{j}=p_{j}, j=1, \cdots, n, \\
& A_{\ell}=I, \quad \ell=1, \cdots, L ; T_{1}(\lambda)=\frac{\lambda^{2}}{2 m}, \lambda \in \mathbb{R}^{n}, \quad T_{\ell}=0, \ell=2, \cdots, L,
\end{aligned}
$$

where $m>0$ is a constant. Then, under the natural identification $\mathcal{K} \otimes L^{2}\left(\mathbb{R}^{n}\right) \cong$ $L^{2}\left(\mathbb{R}^{n} ; \mathcal{K}\right), H$ takes the following form:

$$
H_{\mathrm{S}}:=-\frac{\Delta}{2 m}+\sum_{\ell=1}^{L} B_{\ell} V_{\ell}(q),
$$

where $\Delta:=\sum_{j=1}^{n} D_{j}^{2}$ is the generalized Laplacian acting in $L^{2}\left(\mathbb{R}^{n}\right)$ and $q=\left(q_{1}, \cdots, q_{n}\right)$. This is an $n$-dimensional Schrödinger operator with a $\mathrm{B}(\mathcal{K})$-valued potential. Hence $H_{\mathrm{S}}$ is a generalization of Schrödinger operators with matrix-valued potentials.

Theorem 6.1 Suppose that $H_{\mathrm{S}}$ is self-adjoint. Then, for all $t \in \mathbb{R}$ and $j=1, \cdots, n$,

$$
e^{-i t H_{\mathrm{S}}} D\left(q_{j}\right) \cap D\left(H_{\mathrm{S}}\right)=D\left(q_{j}\right) \cap D\left(H_{\mathrm{S}}\right)
$$

and, for all $\psi \in D\left(q_{j}\right) \cap D\left(H_{\mathrm{S}}\right)$,

$$
q_{j}(t) \psi=q_{j} \psi+\int_{0}^{t} \frac{p_{j}(s)}{m} \psi d s
$$

where

$$
q_{j}(t):=e^{i t H_{\mathrm{S}}} q_{j} e^{-i t H_{\mathrm{S}}}, \quad p_{j}(t):=e^{i t H_{\mathrm{S}}} p_{j} e^{-i t H_{\mathrm{S}}} .
$$

In particular, $q_{j}(t) \psi$ is strongly differentiable in $t \in \mathbb{R}$ and

$$
\frac{d}{d t} q_{j}(t) \psi=\frac{1}{m} p_{j}(t) \psi
$$

Proof. By direct computations, one can easily check that the functions $T_{\ell}$ in the present case satisfy (T.1) and (T.2) with $\partial_{j} T_{1}(\lambda)=\lambda_{j} / m$ and $\partial_{j} T_{\ell}=0, \ell \geq 2$. Also Hypothesis $(\mathrm{H})$ holds. Thus Theorem 5.3 yields the desired result.

As for the momentum operator $p_{j}$, we have the following result:

Theorem 6.2 Assume the following (i)-(iv):

(i) $H_{\mathrm{S}}$ is self-adjoint. 
(ii) For $\ell=1, \cdots L, B_{\ell}$ is bijective and $B_{\ell}^{-1}$ is bounded.

(iii) For each $j=1, \cdots, n$ and $\ell=1, \cdots, L$, there exist positive constants $a_{\ell j}, b_{\ell j}$ such that, for all sufficiently small $|t|(t \in \mathbb{R})$,

$$
\left|V_{\ell}\left(x+t \mathbf{e}_{j}\right)\right|^{2} \leq a_{\ell j}\left|V_{\ell}(x)\right|^{2}+b_{\ell j}, \forall x \in \mathbb{R}^{n} .
$$

(iv) Each $V_{\ell}(\ell=1, \cdots, L)$ is continuously differentiable on $\mathbb{R}^{n}$ and, for $j=1, \cdots, n$ and $\ell=1, \cdots, L$, there exist positive constants $c_{\ell j}, d_{\ell j}$ such that, for all sufficiently small $|t|$,

$$
\left|\partial_{j} V_{\ell}\left(x+t \mathbf{e}_{j}\right)\right|^{2} \leq c_{\ell j}\left|V_{\ell}(x)\right|^{2}+d_{\ell j}, \forall x \in \mathbb{R}^{n} .
$$

Then, for all $t \in \mathbb{R}$ and $j=1, \cdots, n$,

$$
e^{-i t H_{\mathrm{S}}} D\left(p_{j}\right) \cap D\left(H_{\mathrm{S}}\right)=D\left(p_{j}\right) \cap D\left(H_{\mathrm{S}}\right)
$$

and, for all $\psi \in D\left(p_{j}\right) \cap D\left(H_{\mathrm{S}}\right)$,

$$
p_{j}(t) \psi=p_{j} \psi+\int_{0}^{t} F_{j}(s) \psi d s
$$

where

$$
F_{j}(s):=-\sum_{\ell=1}^{L} e^{i s H_{\mathrm{S}}} B_{\ell} \partial_{j} V_{\ell}(q) e^{-i s H_{\mathrm{S}}} .
$$

In particular, $p_{j}(t) \psi$ is strongly differentiable in $t \in \mathbb{R}$ and

$$
\frac{d}{d t} p_{j}(t) \psi=F_{j}(t) \psi
$$

Proof. This follows from Remark 5.1 and the present assumption.

Remark 6.1 The system of differential equations (6.3) and (6.6) gives a mathematically rigorous form of the Heisenberg equation of motion for the pairs $\left(q_{j}(t), p_{j}(t)\right), j=1, \cdots, n$ (a "quantum Newton equation").

\subsection{Relativistic Schrödinger operators}

Let

$$
H_{\mathrm{rel}}:=\sqrt{-\Delta+m^{2}}+\sum_{\ell=1}^{L} B_{\ell} V_{\ell}(q),
$$

a relativistic Schrödinger operator with a $B(\mathcal{K})$-valued potential.

Theorem 6.3 Assume that $V_{\ell} \in L_{\text {loc }}^{2}\left(\mathbb{R}^{n}\right), \ell=1, \cdots, L$, i.e., $\int_{|x| \leq R}\left|V_{\ell}(x)\right|^{2} d x<\infty$ for all $R>0$, and that $H_{\text {rel }}$ is self-adjoint. Then, for all $t \in \mathbb{R}$ and $j=1, \cdots, n$,

$$
e^{-i t H_{\mathrm{rel}}} D\left(q_{j}\right)=D\left(q_{j}\right)
$$


and, for all $\psi \in D\left(q_{j}\right)$,

$$
\widetilde{q}_{j}(t) \psi=q_{j} \psi+\int_{0}^{t} \widetilde{p}_{j}(s)\left(\widetilde{p}(s)^{2}+m^{2}\right)^{-1} \psi d s,
$$

where

$$
\widetilde{q}_{j}(t):=e^{i t H_{\mathrm{rel}}} q_{j} e^{-i t H_{\mathrm{rel}}}, \quad \widetilde{p}_{j}(t):=e^{i t H_{\mathrm{rel}}} p_{j} e^{-i t H_{\mathrm{rel}}} .
$$

In partcular, $\widetilde{q}_{j}(t) \psi$ is strongly differentiable in $t \in \mathbb{R}$ and

$$
\frac{d}{d t} \widetilde{q}_{j}(t) \psi=\widetilde{p}_{j}(t)\left(\widetilde{p}(t)^{2}+m^{2}\right)^{-1} \psi
$$

Proof. In the present case $T_{1}(\lambda)=\sqrt{\lambda^{2}+m^{2}}, T_{\ell}(\lambda)=0, \ell \geq 2, \lambda \in \mathbb{R}^{n}$. Hence (T.1) holds. We have $\partial_{j} T_{1}(\lambda)=\lambda_{j} / \sqrt{\lambda^{2}+m^{2}}, \partial_{j} T_{\ell}=0, \ell \geq 2$. Hence $\partial_{j} T_{\ell}$ is bounded. Moreover, $D\left(q_{j}\right) \cap D\left(H_{\text {rel }}\right)$ is a core for $q_{j}$, since $\mathcal{K} \hat{\otimes} C_{0}^{\infty}\left(\mathbb{R}^{n}\right) \subset D\left(q_{j}\right) \cap D\left(H_{\text {rel }}\right)$ and $C_{0}^{\infty}\left(\mathbb{R}^{n}\right)$ is a core for $q_{j}$. Thus Theorem 5.3-(ii) yields the desired result.

Theorem 6.4 Assume that $H_{\text {rel }}$ is self-adjoint and that conditions (ii)-(iv) in Theorem 6.2 holds. Then, for all $t \in \mathbb{R}$ and $j=1, \cdots, n$,

$$
e^{-i t H_{\mathrm{rel}}} D\left(p_{j}\right) \cap D\left(H_{\mathrm{rel}}\right)=D\left(p_{j}\right) \cap D\left(H_{\mathrm{rel}}\right)
$$

and, for all $\psi \in D\left(p_{j}\right) \cap D\left(H_{\mathrm{rel}}\right)$,

$$
\widetilde{p}_{j}(t) \psi=p_{j} \psi+\int_{0}^{t} F_{j}(s) \psi d s .
$$

In particular, $\widetilde{p}_{j}(t) \psi$ is strongly differentable in $t \in \mathbb{R}$ and

$$
\frac{d}{d t} \widetilde{p}_{j}(t) \psi=F_{j}(t) \psi
$$

Proof. Similar to the proof of Theorem 6.2.

\subsection{Abstract Dirac operators}

In this subsection we consider a class of abstract Dirac operators. Let $\left\{Q_{j}, P_{j} \mid j=\right.$ $1, \cdots, n\}$ be as in Section 5 and $\left\{\Gamma_{j}, B \mid j=1, \cdots, n\right\}$ be a set of bounded self-adjoint operators on $\mathcal{K}$ satisfying

$$
\begin{aligned}
& \left\{\Gamma_{j}, \Gamma_{k}\right\}=2 \delta_{j k}, \quad\left\{\Gamma_{j}, B\right\}=0, \quad j, k=1, \cdots, n, \\
& B^{2}=I
\end{aligned}
$$

where $\{X, Y\}:=X Y+Y X$.

Let $M, V_{\ell}: \mathbb{R}^{n} \rightarrow \mathbb{R}(\ell=1, \cdots, N)$ be Borel measurable functions such that

$$
\hat{E}_{P}\left(\left\{\lambda \in \mathbb{R}^{n}|| M(\lambda) \mid=+\infty\right\}\right)=0, \quad \hat{E}_{Q}\left(\left\{\lambda \in \mathbb{R}^{n}|| V_{\ell}(\lambda) \mid=+\infty\right\}\right)=0 .
$$


Then $M(P)$ and $V_{\ell}(Q)$ are self-adjoint. Let $C_{\ell}$ be a bounded self-adjoint operator on $\mathcal{K}$. Then we define

$$
H_{\mathrm{D}}:=\sum_{j=1}^{n} \Gamma_{j} \otimes P_{j}+B \otimes M(P)+\sum_{\ell=1}^{N} C_{\ell} \otimes V_{\ell}(Q)
$$

As is easily noted, $H_{\mathrm{D}}$ is an abstract version of the usual Dirac operator acting in $L^{2}\left(\mathbb{R}^{3} ; \mathbb{C}^{4}\right)([9$, Chapter 4$])$. Let

$$
H_{0}=\sum_{j=1}^{n} \Gamma_{j} \otimes P_{j}+B \otimes M(P) .
$$

Before proving the self-adjointness of $H_{0}$, we recall the notion of strong anticommutativity on self-adjoint operators:

Definition 6.5 Let $\left\{S_{j}\right\}_{j=1}^{n}$ be a set of self-adjoint operators on a Hilbert space. We say that $\left\{S_{j}\right\}_{j=1}^{n}$ is strongly anticommuting if, for each pair $(j, k)$ with $j \neq k(j, k=1, \cdots, n)$, $e^{i t S_{j}} S_{k} \subset S_{k} e^{-i t S_{j}}, \forall t \in \mathbb{R}$.

Remark 6.2 A posteriori one can show that the definition of the strong anticommutativity is symmetric in $S_{j}, j=1, \cdots, k[6,10]$ and that $e^{i t S_{j}} S_{k}=S_{k} e^{-i t S_{j}}, \forall t \in \mathbb{R}, j \neq k$.

The following theorem is a fundamental result in the theory of strongly anticommuting self-adjoint operators:

Theorem 6.6 (Vasilescu [10]) Let $\left\{S_{j}\right\}_{j=1}^{n}$ be strongly anticommuting on a Hilbert space. Then $T:=\sum_{j=1}^{n} S_{j}$ is self-adjoint and

$$
T^{2}=\sum_{j=1}^{n} S_{j}^{2}
$$

We set

$$
P^{2}:=\sum_{j=1}^{n} P_{j}^{2}
$$

which is a nonnegative self-adjoint operator.

Lemma 6.7 The operator $H_{0}$ is self-adjoint and

$$
\left\|H_{0} \psi\right\|=\left\|I \otimes\left(P^{2}+M(P)^{2}\right)^{1 / 2} \psi\right\|, \quad \psi \in D\left(H_{0}\right) .
$$

Proof. Since $\left\{P_{1}, \cdots, P_{n}, M(P)\right\}$ is a set of strongly commuting self-adjoint operators and (6.13) and (6.14) hold, it follows from a genreral theorem [1, Theorem 3.4] that $\Gamma_{j} \otimes P_{j}$ strongly anticommutes with $\Gamma_{k} \otimes P_{k}(k \neq j)$ and $B \otimes M(P)$. Hence we can apply Vasilescu's theorem (Theorem 6.6) to conclude that $H_{0}$ is self-adjoint and that $H_{0}^{2}=\sum_{j=1}^{n} I \otimes P_{j}^{2}+I \otimes M(P)^{2}$, implying (6.17).

Lemma 6.8 Assume the following (i)-(ii): 
(i) For each $\ell=1, \cdots, N, D\left(\sqrt{P^{2}}\right) \subset D\left(V_{\ell}(Q)\right)$ and there exist positive constant $\varepsilon_{\ell}$ and $b_{\ell}$ such that

$$
\left\|V_{\ell}(Q) \psi\right\| \leq \varepsilon_{\ell}\left\|\sqrt{P^{2}} \psi\right\|+b_{\ell}\|\psi\|, \quad \psi \in D\left(\sqrt{P^{2}}\right)
$$

(ii) The function $M$ is bounded on $\mathbb{R}^{n}$.

Let

$$
\sum_{\ell=1}^{N}\left\|B_{\ell}\right\| \varepsilon_{\ell}<1
$$

Then $H_{\mathrm{D}}$ is self-adjoint with $D\left(H_{\mathrm{D}}\right)=D\left(\sum_{j=1}^{n} \Gamma_{j} \otimes P_{j}\right)=D\left(I \otimes \sqrt{P^{2}}\right)$.

Proof. Let $W:=\sum_{\ell=1}^{N} B_{\ell} V_{\ell}(Q)$. Then, by condition (i), we have for all $\psi \in D(I \otimes$ $\left.\sqrt{P^{2}}\right)=D\left(\sum_{j=1}^{n} \Gamma_{j} \otimes P_{j}\right)$

$$
\|W \psi\| \leq\left(\sum_{\ell=1}^{N}\left\|B_{\ell}\right\| \varepsilon_{\ell}\right)\left\|\left(\sum_{j=1}^{n} \Gamma_{j} \otimes P_{j}\right) \psi\right\|+\left(\sum_{\ell=1}^{N}\left\|B_{\ell}\right\| b_{\ell}\right)\|\psi\|,
$$

where we have used (6.17) with the case $M=0$. Hence, by the Kato-Rellich theorem, $H_{\mathrm{D}}^{\prime}:=\sum_{j=1}^{n} \Gamma_{j} \otimes P_{j}+W$ is self-adjoint with $D\left(H_{\mathrm{D}}^{\prime}\right)=D\left(\sum_{j=1}^{n} \Gamma_{j} \otimes P_{j}\right)=D\left(I \otimes \sqrt{P^{2}}\right)$. Since $H_{\mathrm{D}}=H_{\mathrm{D}}^{\prime}+B \otimes M(P)$ and $B \otimes M(P)$ is a bounded self-adjoint operator under the present assumption, it follows that $H_{\mathrm{D}}$ is self-adjoint with $D\left(H_{\mathrm{D}}\right)=D\left(H_{\mathrm{D}}^{\prime}\right)$.

Theorem 6.9 Suppose that the assumption of Lemma 6.8 holds and that $M$ is continuously differentiable on $\mathbb{R}^{n}$ with $\partial_{j} M$ being bounded on $\mathbb{R}^{n}(j=1, \cdots, n)$. Then, for all $t \in \mathbb{R}$ and $j=1, \cdots, n$,

$$
e^{-i t H_{\mathrm{D}}} D\left(I \otimes Q_{j}\right)=D\left(I \otimes Q_{j}\right)
$$

and, for all $\psi \in D\left(I \otimes Q_{j}\right)$,

$$
e^{i t H_{\mathrm{D}}}\left(I \otimes Q_{j}\right) e^{-i t H_{\mathrm{D}}} \psi=I \otimes Q_{j} \psi+\int_{0}^{t} e^{i s H_{\mathrm{D}}}\left[\Gamma_{j} \otimes I+B \otimes\left(\partial_{j} M\right)(P)\right] e^{-i s H_{\mathrm{D}}} \psi d s .
$$

Proof. We apply Theorem 5.3 to the following case:

$$
\begin{aligned}
& L:=\max \{n+1, N\}, \\
& A_{\ell}=\left\{\begin{array}{ll}
\Gamma_{\ell} \quad ; \ell=1, \cdots, n, \\
B \quad ; \ell=n+1, \\
A_{\ell}=0 ; \quad \ell>n+1
\end{array}, \quad T_{\ell}(\lambda)=\left\{\begin{array}{ll}
\lambda_{\ell} & ; \ell=1, \cdots, n, \\
M(\lambda) & ; \ell=n+1, \\
T_{\ell}=0 & ; \ell>n+1
\end{array},\right.\right. \\
& B_{\ell}= \begin{cases}C_{\ell} \quad \ell=1, \cdots, N, \\
0 \quad ; \ell>N\end{cases}
\end{aligned}
$$

We also note that $D\left(I \otimes Q_{j}\right) \cap D\left(\sum_{j=1}^{n} \Gamma_{j} \otimes P_{j}\right)$ is a core of $I \otimes Q_{j}$, since $\left\{Q_{j}, P_{j} \mid j=1, \cdots, n\right\}$ is a Weyl representation of CCR. Thus we obtain the desired result from Theorem 5.3-(ii).

Remark 6.3 One can also obtain a result on $I \otimes P_{j}$. But we omit writing it down. 


\section{Acknowledgment}

This work is supported by the Grant-In-Aid 17340032 for scientific research from the Japan Society for the Promotion of Science (JSPS).

\section{References}

[1] A. Arai, Characterization of anticommutativity of self-adjoint operators in connection with Clifford algebra and applications, Integr. Equat. Oper. Th. 17 (1993), 451-463.

[2] H. L. Cycon, R. G. Froese, W. Kirsch and B. Simon, Schrödinger Operators, Springer, Berlin, Heidelberg, 1987.

[3] V. Georgescu and C. Gérard, On the virial theorem in quantum mechanics, Commun. Math. Phys. 208(1999), 275-281.

[4] T. Kato, Perturbation Theory for Linear Operators, Second Edition, Springer, Berlin Heidelberg, 1976.

[5] J. von Neumann, Die Eindeutigkeit der Schrödingerschen Operatoren, Math. Ann. 104 (1931), 570-578.

[6] S. Pedersen, Anticommuting self-adjoint operators, J. Funct. Anal. 89 (1990), 428443.

[7] M. Reed and B. Simon, Methods of Modern Mathematical Physics I: Functional Analysis, Academic Press, New York, 1972.

[8] M. Reed and B. Simon, Methods of Modern Mathematical Physics II: Fourier Analysis, Self-adjointness, Academic Press, New York, 1975.

[9] B. Thaller, The Dirac Equation, Springer, Berlin, Heidelberg, 1992.

[10] F.-H. Vasilescu, Anticommuting self-adjoint operators, Rev. Roum. Math. Pures et Appl. 28 (1983), 77-91. 